\title{
Exact expression for the effective acoustics of patchy-saturated rocks
}

\author{
Bouko Vogelaar ${ }^{1}$, David Smeulders ${ }^{2}$, and Jerry Harris ${ }^{1}$
}

\begin{abstract}
Seismic effects of a partially gas-saturated subsurface have been known for many years. For example, patches of nonuniform saturation occur at the gas-oil and gas-water contacts in hydrocarbon reservoirs. Open-pore boundary conditions are applied to the quasi-static Biot equations of poroelasticity to derive an exact analytic expression of the effective bulk modulus for partially saturated media with spherical gas patches larger than the typical pore size. The pore fluid and the rock properties can have different values in the central sphere and in the surrounding region. An analytic solution prevents loss of accuracy from ill-conditioned equations as encountered in the numerical solution for certain input. For a sandstone saturated with gas and water, we found that the P-wave velocity and attenuation in conventional models differ as much as $15 \%$ from the exact solution at seismic frequencies. This makes the use of present exact theory necessary to describe patchy saturation, although (more realistic) complex patch shapes and distributions were not considered. We found that, despite earlier corrections, the White conventional model does not yield the correct low-frequency asymptote for the attenuation.
\end{abstract}

\section{INTRODUCTION}

When a porous rock is saturated by a mixture of two fluids, patchy saturation refers to the case in which the saturation scale is large enough so that the wave-induced pore-pressure changes cannot equilibrate during a seismic period (Mavko and Mukerji, 1998). Patches of nonuniform saturation occur at the gas-oil and gas-water contacts in hydrocarbon reservoirs. During production, the pressure decrease might lead to nucleation and the diffusive formation of free gas pockets. The contact between gas and water in reservoirs is not necessarily sharp, and typically a transition zone exists between full gas and full water saturation.
Seismic low-frequency effects of partially gas-saturated hydrocarbon reservoirs have been known for many years (e.g., Castagna et al., 2003; Chapman et al., 2006; Goloshubin et al., 2006, and references therein). For example, high-resolution surveys make it feasible to detect pockets of unswept reserves and to monitor the progress of enhanced recovery by gas and water injection (Helle et al., 2003), and the inclusion of P-wave velocity dispersion in interpreting well $\log$ data of partially saturated sediments can be used to identify free gas and relate surface seismic data to synthetic seismograms (Lee and Collett, 2009). The real-data observations of Saenger et al. (2009) are consistent with a partially saturated hydrocarbon reservoir model in which poroelastic effects caused by wave-induced fluid flow and oscillations of different fluid phases can modify the omnipresent seismic background spectrum.

Laboratory observations have also demonstrated the effect of partial saturation on acoustic velocities (e.g., Winkler and Nur, 1979; Murphy, 1982; Paffenholz and Burkhardt, 1989; Knight et al., 1998) and are supported by X-ray computer tomography images of the patch distribution (e.g., Cadoret et al., 1995; Monsen and Johnstad, 2005; Lebedev et al., 2009; Toms-Stewart et al., 2009) and numerical simulations (e.g., Carcione et al., 2003; Masson and Pride, 2007; Picotti et al., 2007; Wenzlau and Müller, 2009).

Wave-induced fluid flow effects in the fluid are modeled by isolated spherical gas patches in the liquid saturating a homogeneous matrix, as first proposed by White (1975). Since then, significant progress has been made by considering various patch distributions and flow regimes (White et al., 1975; Dutta and Odé, 1979a, 1979b; Norris, 1993; Gelinsky et al., 1998; Johnson, 2001; Müller and Gurevich, 2004, 2005; Müller et al., 2008; Gurevich et al., 2009; Picotti et al., 2010). More references for this topic are found in Toms et al. (2006). When a gas pocket is subjected to the macroscopic pressure field of a compressional seismic wave (i.e., on a length scale much larger than the size of the inhomogeneity), the pocket will contract and expand. These oscillations generate waves on the mesoscale (i.e., on the length scale of the inhomogeneity), which consume energy from the seismic wave, causing intrinsic attenuation.

Patchy-saturation theories apply to a nonrigid porous medium fully saturated by a fluid that contains gas pockets (radius $a$ ) larger than

Manuscript received by the Editor 23 November 2009; revised manuscript received 24 March 2010; published online 6 August 2010.

${ }^{1}$ Stanford University, Department of Geophysics, Stanford, California, U.S.A. E-mail: vogelaar@ @stanford.edu; jerry.harris@ stanford.edu.

${ }^{2}$ Delft University of Technology, Department of Geotechnology, Delft, The Netherlands. E-mail: d.m.j.smeulders@ citg.tudelft.nl. (C) 2010 Society of Exploration Geophysicists. All rights reserved. 
the typical pore size; see Figure 1. The interaction among the individual gas pockets is neglected by defining a liquid influence shell (radius $b$ ) surrounding each pocket. The gas fraction is $s_{a}=(a / b)^{3}$. The radius $b$ is chosen so that the volume of the sphere $\frac{4}{3} \pi b^{3}$ equals the volume of the unit cell of the cubic lattice.

The external pressure field is assumed to be spatially homogeneous at the scale of the inhomogeneity, and the effective (macroscopic) bulk modulus can be obtained by considering a representative volume comprising a single gas pocket and a liquid shell surrounding the pocket. The effective bulk modulus $K(\omega)$ can then be deduced via its definition:

$$
K(\omega)=-\frac{b}{3 u(b)} p_{\mathrm{e}},
$$

where $u(b)$ is the complex-valued radial solid displacement at the outer boundary of the unit cell. Solving the Biot (1956a) equations yields the solid displacement as a function of the applied pressure $p_{\mathrm{e}}$ and hence the effective bulk modulus of the representative volume.

Once the effective bulk modulus is obtained, the velocity $c$ $=\operatorname{Re}\left(k_{1}\right) / \omega$ and attenuation (inverse quality factor) $Q^{-1}$ $=2 \operatorname{Im}\left(k_{1}\right) / \operatorname{Re}\left(k_{1}\right)$ of the seismic wave are computed using the effective complex wavenumber

$$
k_{1}(\omega)=\omega \sqrt{\rho /\left(K(\omega)+\frac{4}{3} \mu\right)}
$$

with shear modulus $\mu$. Subscript 1 describes the fast wave on the macroscale, and $\rho$ is the total density $(1-\phi) \rho_{\mathrm{s}}+\phi\left(\left(1-s_{a}\right) \rho_{\mathrm{f} b}\right.$ $+s_{a} \rho_{\mathrm{f} a}$ ), where $\rho_{\mathrm{f} a, b}$ denotes the gas (subscript $a$ ) or liquid (subscript b) density.

In this paper, we derive the exact analytic solution to patchy saturation in the quasi-static Biot (1956a) context for spherical gas pockets. Although the governing equations were already given by Johnson (2001), no analytic solution was yet available. We obtain the exact solution by solving the boundary value problem of an 8

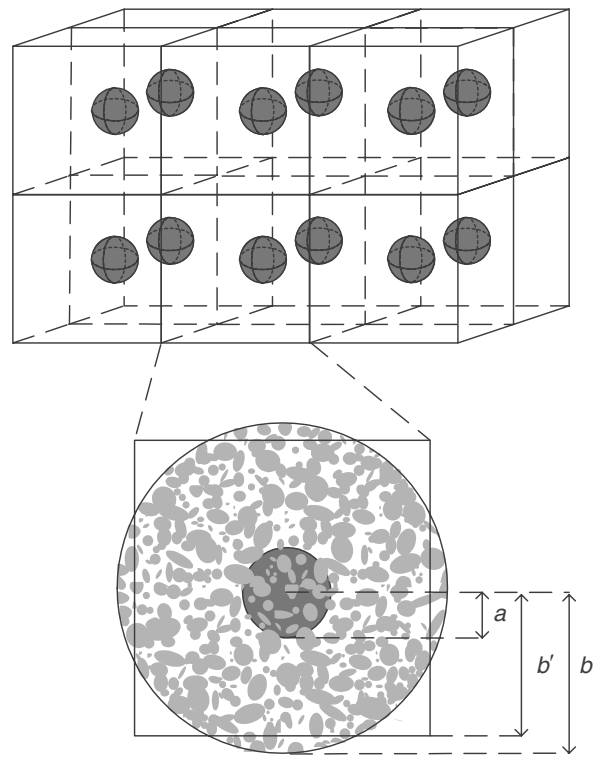

Figure 1. Geometry of a cubic lattice of periodic spherical gas pockets with radius $a$, separated by distance $2 b^{\prime}$. Each gas pocket is surrounded by a liquid shell with radius $b$, so that the volume of the cube equals the volume of the sphere $V_{b^{\prime}}=V_{b}$. $\times 8$ system analytically. Johnson (2001) solved this system numerically, and he introduced a generalized dynamic bulk modulus.

We compare our analytic quasi-static solution with the numerical solution of Dutta and Odé (1979a) and the widely adopted analytic quasi-static solution of White et al. (1975), including the modifications by Dutta and Seriff (1979). Following Johnson (2001), we also compare our results with those using the generalized dynamic bulk modulus approach. Dutta and Odé (1979a) computed the behavior over the full frequency domain, but they encountered numerical loss of accuracy at high frequencies. An analytic solution implicitly prevents errors from the numerical inversion of ill-conditioned equations, as also encountered by Dutta and Odé (1979a).

\section{QUASI-STATIC PATCHY THEORY}

\section{Concentric spheres geometry}

Consider first the response of a homogeneous sample fully saturated with a single fluid to a uniform compressive stress. We presume that the frequency is low enough that the Biot (1956a, 1956b) theory is in its low-frequency limit (quasi-static case). Thus the fast compressional and shear waves are nondispersive and nonattenuating, whereas the slow compressional wave is diffusive in character. The requirement is $\omega \ll \omega_{\mathrm{B}}$, where the Biot crossover frequency is $\omega_{\mathrm{B}}=\phi \eta /\left(k_{0} \alpha_{\infty} \rho_{\mathrm{f}}\right)$. The rock properties are porosity $\phi$, permeability $k_{0}$, and tortuosity $\alpha_{\infty}$; the fluid properties are density $\rho_{\mathrm{f}}$ and shear viscosity $\eta$.

The starting equations are essentially those of the Biot (1956a) theory at low frequencies by setting to zero all higher order inertial terms and by taking the dynamic permeability equal to its steadystate value $k_{0}$ (Norris, 1993; Johnson, 2001). Adopting an $\exp (i \omega t)$ dependence for all relevant quantities, the quasi-static Biot equations are

$$
\nabla \cdot \hat{\tau}=0
$$

$$
\frac{k_{0}}{\eta \phi} \nabla \hat{p}=i \omega(\hat{\mathbf{u}}-\hat{\mathbf{U}}),
$$

where $\mathbf{u}$ and $\mathbf{U}$ are the solid and fluid displacement, respectively. The accent circumflex over a field variable (displacement, stress, and pressure) denotes small variations of that variable. The accent circumflex over the dependencies of these field variables (such as strain) is omitted. Using the summation convention, the total stress $\tau_{i j}$ (solid plus fluid phases) and pore fluid pressure $p$ in terms of the solid and fluid strains $e_{i j}=\nabla \cdot \hat{\mathbf{u}}$ and $\varepsilon_{i j}=\nabla \cdot \hat{\mathbf{U}}$ are, in the case of isotropic materials (Johnson, 2001),

$$
\begin{gathered}
\tau_{i j}=\left[(P+Q-2 \mu) e_{k k}+(Q+R) \epsilon_{k k}\right] \delta_{i j}+2 \mu e_{i j}, \\
-\phi p=Q e_{k k}+R \epsilon_{k k} .
\end{gathered}
$$

Explicit expressions of the poroelastic coefficients are given in terms of the bulk moduli of the pore fluid, the solid, and the matrix $K_{\mathrm{f}, \mathrm{s}, \mathrm{m}}$, respectively, as (Biot and Willis, 1957)

$$
\begin{aligned}
& P=\frac{\phi K_{\mathrm{m}}+(1-\phi) K^{\prime}}{\phi^{\prime}}+\frac{4}{3} \mu, \\
& Q=\frac{\phi K^{\prime}}{\phi^{\prime}},
\end{aligned}
$$




$$
R=\frac{\phi^{2} K_{\mathrm{f}}}{\phi^{\prime}}
$$

where $\phi^{\prime}=\phi+K^{\prime} / K_{\mathrm{s}}$ and $K^{\prime}=K_{\mathrm{f}}(\alpha-\phi)$ are auxiliary parameters and $\alpha=1-K_{\mathrm{m}} / K_{\mathrm{s}}$ is the so-called Biot-Willis coefficient. For later use, we define the Biot-Gassmann modulus

$$
K_{\mathrm{BG}}=H-\frac{4}{3} \mu
$$

in terms of the static compressional-wave modulus $H=P+2 Q$ $+R$ and the shear modulus $\mu$.

The spherically symmetrical solutions to equations 3 and 4 obey the following equations (Johnson, 2001):

$$
\frac{\partial}{\partial r}\left[(P+Q)\left(\frac{\partial u}{\partial r}+\frac{2 u}{r}\right)+(R+Q)\left(\frac{\partial U}{\partial r}+\frac{2 U}{r}\right)\right]=0
$$

and

$$
\frac{i \omega \eta \phi^{2}}{k_{0}}(U-u)=\frac{\partial}{\partial r}\left[Q\left(\frac{\partial u}{\partial r}+\frac{2 u}{r}\right)+R\left(\frac{\partial U}{\partial r}+\frac{2 U}{r}\right)\right],
$$

where $u$ and $U$ are the radial displacements of the solid and the fluid, respectively.

Following Johnson (2001), there are two kinds of solutions to the above equations: (1) Solutions for which the fluid motion is locked on to the solid's motion, $u(r) / U(r)=1$, are linear combinations of $r$ and $r^{-2}$. These are low-frequency fast compressional-wave solutions. (2) Solutions for which $u(r) / U(r)=-(P+Q) /(Q+R)$ are linear combinations of spherical Bessel functions $j_{1}\left(k_{2} r\right)$ and $n_{1}\left(k_{2} r\right)$, where $k_{2}(=\sqrt{-i \omega / D})$ is the wavenumber of the slow compressional Biot wave. For these solutions, the fluid and solid move out-of-phase, and the relevant quantities obey a diffusion equation with the slow wave diffusivity given by

$$
D=\frac{k_{0}}{\eta \phi^{2}} \frac{P R-Q^{2}}{H} .
$$

The general solution for the radial direction is therefore written as (Johnson, 2001)

$$
\begin{aligned}
u(r)= & A r+\frac{B}{r^{2}}+(Q+R)\left[F j_{1}\left(k_{2} r\right)+G n_{1}\left(k_{2} r\right)\right], \\
U(r)= & A r+\frac{B}{r^{2}}-(P+Q)\left[F j_{1}\left(k_{2} r\right)+G n_{1}\left(k_{2} r\right)\right], \\
p(r)= & -\frac{3(Q+R)}{\phi} A+\frac{\left(P R-Q^{2}\right)}{\phi} k_{2}\left[F j_{0}\left(k_{2} r\right)\right. \\
& \left.+G n_{0}\left(k_{2} r\right)\right], \\
\tau(r)= & 3 K_{\mathrm{BG}} A-\frac{4 \mu}{r^{3}} B-\frac{4 \mu(Q+R)}{r}\left[F j_{1}\left(k_{2} r\right)\right. \\
& \left.+G n_{1}\left(k_{2} r\right)\right] .
\end{aligned}
$$

The above equations are general and apply to both the gas sphere and the liquid shell. The quantities $P, Q, R, K_{\mathrm{BG}}$, and $k_{2}(\omega)$ have dif- ferent values in the two regions because they are functions of the pore fluid and the rock properties. The above equation set 12 was solved numerically by Johnson (2001), but we show here that the exact analytic solution is readily available.

\section{Exact analytic solution}

The quasi-static solution to patchy saturation, equation 1 , is found by applying the Biot (1956a) theory to the geometry of Figure 1 using the appropriate boundary conditions. There are eight arbitrary constants in the above equation set 12: $A, B, F$, and $G$ in each of the two regions. The requirement that the particular solution be finite at $r=0$ implies $B_{a}=0$ and $G_{a}=0$. The remaining six unknowns are found from the continuity of solid displacement $\mathbf{u}$ and fluid displacement $\mathbf{U}$, pressure $p$ and total stress $\tau$ at $r=a$, and the condition that at $r=b$ the external pressure $p_{\mathrm{e}}$ is applied at a sealed pore boundary (Deresiewicz and Skalak, 1963; Dutta and Odé, 1979a; Johnson, 2001):

$$
\begin{aligned}
& u_{a}(a)=u_{b}(a), \\
& U_{a}(a)=U_{b}(a), \\
& p_{a}(a)=p_{b}(a), \\
& \tau_{a}(a)=\tau_{b}(a), \\
& u_{b}(b)=U_{b}(b), \\
& \tau_{b}(b)=-p_{\mathrm{e}} .
\end{aligned}
$$

Analogous boundary conditions are used by, e.g., Berryman and Thigpen (1985), Taylor and Knight (2003), and Ciz and Gurevich (2005) in related problems. By solving this set, the field variables are determined uniquely everywhere. The sealed pore condition at the outer boundary, equation 17 , gives

$$
G_{b}=-F_{b} \frac{j_{1}\left(k_{2 b} b\right)}{n_{1}\left(k_{2 b} b\right)} \text {. }
$$

This means that all expressions (equation set 12) in the liquid region can be rewritten using the auxiliary parameter

$$
f_{\ell}=j_{\ell}\left(k_{2 b} a\right)-n_{\ell}\left(k_{2 b} a\right) \frac{j_{1}\left(k_{2 b} b\right)}{n_{1}\left(k_{2 b} b\right)},
$$

where $\ell=0,1$ is the order of the spherical Bessel function. Subtraction of the solid and fluid continuity equations 13 and 14 at $r=a$ leads to

$$
F_{a} j_{1}\left(k_{2 a} a\right)=F_{b} f_{1} \frac{H_{b}}{H_{a}},
$$

so that the solid continuity equation 13 becomes

$$
A_{a} a=A_{b} a+\frac{B_{b}}{a^{2}}+F_{b} f_{1} N
$$

with 


$$
N=\left(R_{b}+Q_{b}\right)-\left(R_{a}+Q_{a}\right) \frac{H_{b}}{H_{a}} .
$$

The continuity of total stress and pore pressure at the interface between the regions now yields that

$$
\begin{gathered}
\frac{3 a}{4 \mu} A_{a} K_{\mathrm{BG} a}=\frac{3 a}{4 \mu} A_{b} K_{\mathrm{BG} b}-\frac{B_{b}}{a^{2}}-F_{b} f_{1} N, \\
-3 A_{a}\left(R_{a}+Q_{a}\right)+3 A_{b}\left(R_{b}+Q_{b}\right) \\
=F_{b}\left(P_{b} R_{b}-Q_{b}^{2}\right) k_{2 b} f_{0}(1-h),
\end{gathered}
$$

where we introduced another auxiliary parameter

$$
h=\frac{\eta_{a}}{\eta_{b}} \frac{D_{a}}{D_{b}} \frac{k_{2 a}}{k_{2 b}} \frac{j_{0}\left(k_{2 a} a\right)}{j_{1}\left(k_{2 a} a\right)} \frac{f_{1}}{f_{0}}
$$

where the slow wave diffusivity $D$ in each region is given by equation 11 . Adding equations 22 and 24 yields the surprisingly simple relationship

$$
A_{a} H_{a}=A_{b} H_{b} .
$$

The combination of equations 22,25 , and 27 yields

$$
B_{b}=-b^{3} A_{b} g
$$

with

$$
g=s_{a}\left(1-\frac{H_{b}}{H_{a}}+\frac{3 N^{2}}{P_{b} R_{b}-Q_{b}^{2}} \frac{f_{1}}{f_{0}} \frac{1}{k_{2 b} a} \frac{1}{1-h}\right) .
$$

The last boundary condition (equation 18) finally implies

$$
3 A_{b} K_{\mathrm{BG} b}-\frac{4 \mu}{b^{3}} B_{b}=-p_{\mathrm{e}}
$$

so that with equation 28 this becomes

$$
A_{b}=-\frac{p_{\mathrm{e}}}{3 K_{\mathrm{BG} b}+4 \mu g} .
$$

The solid displacement at the outer boundary is $u(b)=A_{b} b(1-g)$, and with equation 1 , the exact expression for the quasi-static bulk modulus $K(\omega)$ is

$$
K(\omega)=\frac{K_{\mathrm{BG} b}+\frac{4}{3} \mu g}{1-g} .
$$

All information about the presence of gas in the liquid phase is solely captured in parameter $g$. It is clear from equations 29 and 32 that, in the case of complete liquid saturation, $s_{a}=0$ and $g=0$, so that $K(\omega)$ reduces to $K_{\mathrm{BG} b}$. For complete gas saturation, $s_{a}=1$ or $a=b$, so that $f_{1}=0$ in equation 20. This means that $g=1-H_{\mathrm{b}} / H_{\mathrm{a}}$, and using equation $8, K(\omega)$ reduces to $K_{\mathrm{BG} a}$. Equation 32 is an exact analytic expression for partially saturated media, which reduces to the BiotGassmann limit in the case of full saturation by a single fluid.

Expressions 29 and 32 comprise the combined effect of fast and slow compressional-wave contributions. In the lower (static) and upper (no-flow) bounds of $K(\omega)$, there are no effects of pressure diffusion (i.e., slow wave effects). These bounds are given in Appendices $\mathrm{A}$ and $\mathrm{B}$. The slow wave contributions associated with viscous dissipation due to the relative fluid-solid movement occur only in the intermediate frequency regime through the wavenumbers $k_{2 a}$ and $k_{2 b}$.

\section{Comparison of the exact solution with other solutions}

The parameter set of Table 1 is chosen to show the effective bulk modulus as a function of frequency in Figure 2. The gas fraction is 0.1 and $b=0.1 \mathrm{~m}$. The results are shown for four calculations: (1) The present exact analytic quasi-static solution (solid curve); (2) the analytic quasi-static solution (dotted curve) of White (1975), including the corrections by Dutta and Seriff (1979); (3) the exact numerical solution (dots) in the full-Biot context by Dutta and Odé (1979a); and (4) the generalized analytic quasi-static calculations (dashed curve) of Johnson (2001). The low- and high-frequency limiting moduli, Biot-Gassmann-Wood modulus $K_{\mathrm{BGW}}$ and Biot-GassmannHill modulus $K_{\mathrm{BGH}}$, respectively, are given by dashed-dotted lines.

As long as the frequency is low enough, the exact analytic and numerical full-frequency solutions are identical. The computation of the effective bulk modulus of the representative volume makes sense only if the frequency is low enough so that the wavelengths of the fast compressional and shear waves are large compared to the dimensions of the pockets and their mutual distance (Johnson, 2001). This means that the mesoscopic condition $\omega \ll \omega_{r}$ should hold where $\omega_{r} \sim c_{\mathrm{S}} / b$, in which $c_{\mathrm{S}}$ is the speed of the shear wave in the region of the liquid. When the wavelength of the shear wave becomes of the same order as the patch size $b$, resonance occurs in the full-frequency solution. The frequency at which the medium becomes extremely compliant is $\omega_{r} / 2 \pi=1.4 \mathrm{kHz}$. On increasing the frequency, the medium oscillates out-of-phase with respect to the applied pressure $p_{\mathrm{e}}$ (antiresonance), and fluctuating values of the bulk modulus occur in the full-frequency solution.

Resonances and antiresonances occur in coupled systems when one (or more) of the systems is finite in size (Morse and Ingard, 1968). At the antiresonance frequency, no incident energy of the external (macroscopic) wavefield is radiated from the medium, so that the medium behaves as though it were rigid and very high values of the bulk modulus are reached. Similar resonance phenomena (scattering) are seen for the full-frequency solutions in analogous problems (e.g., Shapiro and Müller, 1999; Jocker et al., 2004; and Vogelaar and Smeulders, 2007).

The analytic structure of $K(\omega)$ allows investigation of the real and imaginary parts or the absolute value and phase. Both approaches exhibit their own distinct characteristic transition frequency, which

Table 1. Constituent properties of the partially saturated sandstone rock with a relatively weak frame (Johnson, 2001).

\begin{tabular}{lccll}
\hline & Matrix & Grains & Water & Gas \\
\hline Density $\rho\left[\mathrm{kg} / \mathrm{m}^{3}\right]$ & & $2.65 \cdot 10^{3}$ & $1.0 \cdot 10^{3}$ & 1.0 \\
Bulk modulus $K[\mathrm{~Pa}]$ & $2.637 \cdot 10^{9}$ & $35.0 \cdot 10^{9}$ & $2.25 \cdot 10^{9}$ & $1.0 \cdot 10^{5}$ \\
Viscosity $\eta[\mathrm{Pa} \cdot \mathrm{s}]$ & & & $1.0 \cdot 10^{-3}$ & $1.0 \cdot 10^{-5}$ \\
Porosity $\phi[-]$ & 0.284 & & & \\
Permeability $k_{0}\left[\mathrm{~m}^{2}\right]$ & $1.0 \cdot 10^{-13}$ & & & \\
Shear modulus $\mu[\mathrm{Pa}]$ & $1.740 \cdot 10^{9}$ & & & \\
\hline
\end{tabular}


is commonly referred to as the relaxation frequency because it characterizes the transition from the relaxed (drained) to the unrelaxed (undrained) response as the frequency increases.

The deviations of the models by White (1975) and Johnson (2001) from our exact analytic solution occur around the transition frequency $\omega_{c} \approx D_{b} / b^{2}$ (Pride et al., 2004). Using equation 11, we obtain $\omega_{c} / 2 \pi=5 \mathrm{~Hz}$. At this transition frequency, the Biot slow wave diffusion length equals the characteristic length of the inhomogeneity (Gurevich and Lopatnikov, 1995). This equation indicates that the mesoscopic loss mechanism moves toward lower frequencies with increasing viscosity and decreasing permeability. This behavior is opposed to the Biot (1956a, 1956b) relaxation mechanism (global flow). Note that the peak frequency in the Johnson model is identical to our exact frequency value, whereas the one in the White model is at lower frequencies. The difference depends on the rock and fluid properties and increases with the increasing gas fraction.

Johnson (2001) and Pride et al. (2004) connect the high- and lowfrequency asymptotes of their functions by a simple frequency function. The exact expression of $K(\omega)$ allows one to define the exact transition frequency (where $\operatorname{Im}\{K\}$ is maximum or $\operatorname{Re}\{K\}$ has its inflection point) by setting $d \operatorname{Im}\{K\} / d \omega=0$ or $d^{2} \operatorname{Re}\{K\} / d \omega^{2}=0$. Another choice is where the phase value of $K$ is maximum or where the absolute value of $K$ has its inflection point. Due to the complex nature of the spherical Bessel functions, it is not straightforward to retrieve a simple exact expression for $\omega_{c}$. We do note, however, that for a wide range of rock and fluid properties and fractional volumes, $K_{\mathrm{fb}} k_{0} / 2 \pi \eta_{b} \phi(b-a)^{2}$ gives a good approximation of the frequency where the imaginary part of $K$ is maximum. Similar relations are, e.g., given by Pride et al. (2002) and Carcione et al. (2003). The exact transition frequency provides the exact maximum level of attenuation. For a reservoir rock with alternating gas and water saturation, Quintal et al. (2009) recently found that the maximum level of attenuation can be approximated by only five parameters $\left(K_{\mathrm{m}}, K_{\mathrm{s}}, \mu, K_{\mathrm{f} b}\right.$, and $\phi$ ).

\section{VELOCITY AND ATTENUATION EXAMPLES}

To illustrate the usefulness of our exact expression, we show the accuracy of velocity and attenuation predicted by the other three patchy models (corrected White, full Dutta-Odé, and generalized Johnson). We use the constituent properties of Table 1 with $b$ $=0.1 \mathrm{~m}$. In Figure 3, the gas fraction is 0.1 , and in Figure 4 the gas fraction is 0.5 .

Figure 3 a shows the introduced error in $\mathrm{P}$-wave velocity as a function of frequency. The absolute error is the difference between our exact velocity and the velocity calculated by each model. For a gas fraction of $10 \%$, the underestimation in the velocity is $20 \mathrm{~m} / \mathrm{s}$ at $100 \mathrm{~Hz}$ for the corrected White model and $15 \mathrm{~m} / \mathrm{s}$ for the Johnson model. At lower frequencies, both models overestimate the velocity (e.g., $10 \mathrm{~m} / \mathrm{s}$ at $20 \mathrm{~Hz}$ for the White model). The full-frequency Dutta-Odé model is identical to our quasi-static result below $100 \mathrm{~Hz}$.

Figure $3 \mathrm{~b}$ shows the introduced error in the $\mathrm{P}$-wave attenuation. Here, the error is relative; i.e., it is the difference between the exact and approximate attenuation relative to the exact attenuation value. The most striking result of present analysis is that, despite the staticvelocity corrections by Dutta and Seriff (1979), the White model does not yield the correct low-frequency value for the attenuation. For a gas fraction of 0.1 , the attenuation is overestimated by about $3 \%$ for low frequencies $(<10 \mathrm{~Hz})$. For higher frequencies, the rela- tive error in attenuation ranges from $-7 \%$ at $60 \mathrm{~Hz}$ to $+12 \%$ at 4 $\mathrm{kHz}$ for the White model. For the generalized Johnson model, the introduced errors in attenuation are between $-4 \%$ at $40 \mathrm{~Hz}$ and $+8 \%$ at $250 \mathrm{~Hz}$.

The errors in velocity and attenuation from the White and generalized Johnson solutions depend on the gas fraction. In Figure 4a, the gas fraction is 0.5 , and the maximum error in velocity is $-18 \mathrm{~m} / \mathrm{s}$ for the White model and $+11 \mathrm{~m} / \mathrm{s}$ for the generalized Johnson model. The Dutta-Odé model is accurate below $100 \mathrm{~Hz}$. The attenuation predicted by the White model, Figure $4 \mathrm{~b}$, is almost $20 \%$ too high at low frequencies $(<100 \mathrm{~Hz})$.

For very low or very high gas fractions, the calculations show that the error in predicted velocity by the White and the generalized
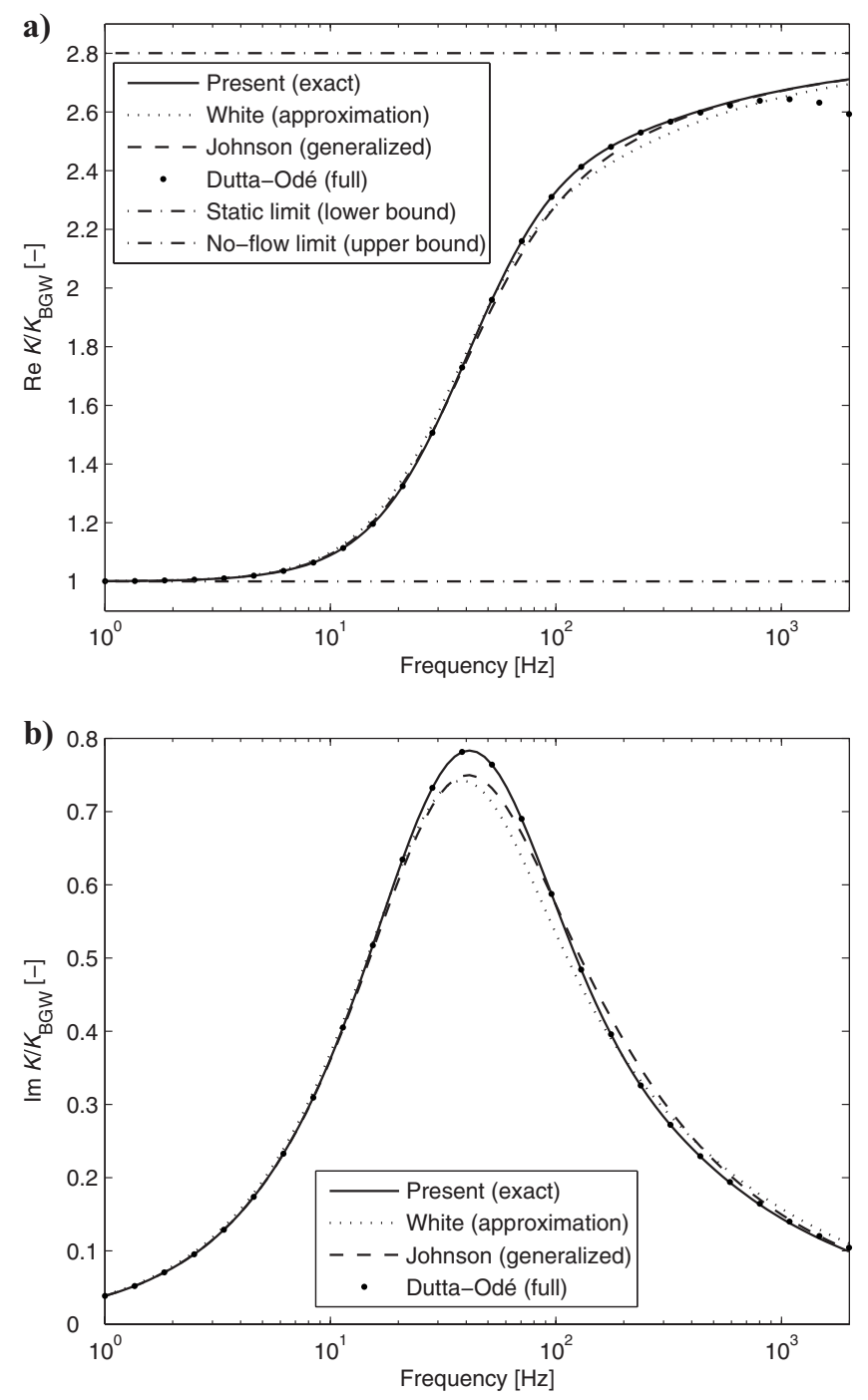

Figure 2. Effective bulk modulus as a function of frequency. (a) Real part of $K / K_{\mathrm{BGW}}$. (b) Imaginary part of $K / K_{\mathrm{BGW}}$. The solid curve is the present exact analytic solution in the quasi-static context (equation 32). The dotted curve is the analytic solution by White (1975), as corrected by Dutta and Seriff (1979). The dashed curve is the generalized analytic solution by Johnson (2001). The dots are the exact numerical solutions in the full Biot context by Dutta and Odé (1979a). The lower and upper horizontal dashed-dotted lines are the limiting moduli $K_{\mathrm{BGW}}$ and $K_{\mathrm{BGH}}$, equations A-6 and B-2. The input values are from Table $1 ; s_{a}=0.1$ and $b=0.1 \mathrm{~m}$. 
Johnson models is less than $4 \mathrm{~m} / \mathrm{s}$. However, for, say, $s_{a}=0.001$, the attenuation predicted by the White model is $20 \%$ too high at $5 \mathrm{kHz}$ and $15 \%$ too low at $700 \mathrm{~Hz}$ for the generalized Johnson model. For $s_{a}=0.9$, the predicted attenuation is more than $40 \%$ too high for the White model below $1 \mathrm{kHz}$ (i.e., in the entire seismic range) and more than $15 \%$ too high for the generalized Johnson model from 30 to $1000 \mathrm{~Hz}$. Indeed, for high gas fractions $\left(s_{a}>0.52\right)$, the gasfilled spheres in the cubical lattice interact, and we might consider water-filled spheres surrounded by gas. The White calculations then predict the attenuation also to be more than $40 \%$ too high below $100 \mathrm{~Hz}$.

White (1975) also considers the case in which the central sphere is saturated with a very compressible gas. For $K_{\mathrm{f} a} \rightarrow 0$, equation 29 becomes

$$
g^{*}=s_{a}\left(1-\frac{H_{b}}{K_{\mathrm{m}}+\frac{4}{3} \mu}+\frac{3\left(R_{b}+Q_{b}\right)^{2}}{P_{b} R_{b}-Q_{b}^{2}} \frac{f_{1}}{f_{0}} \frac{1}{k_{2 b} a}\right),
$$
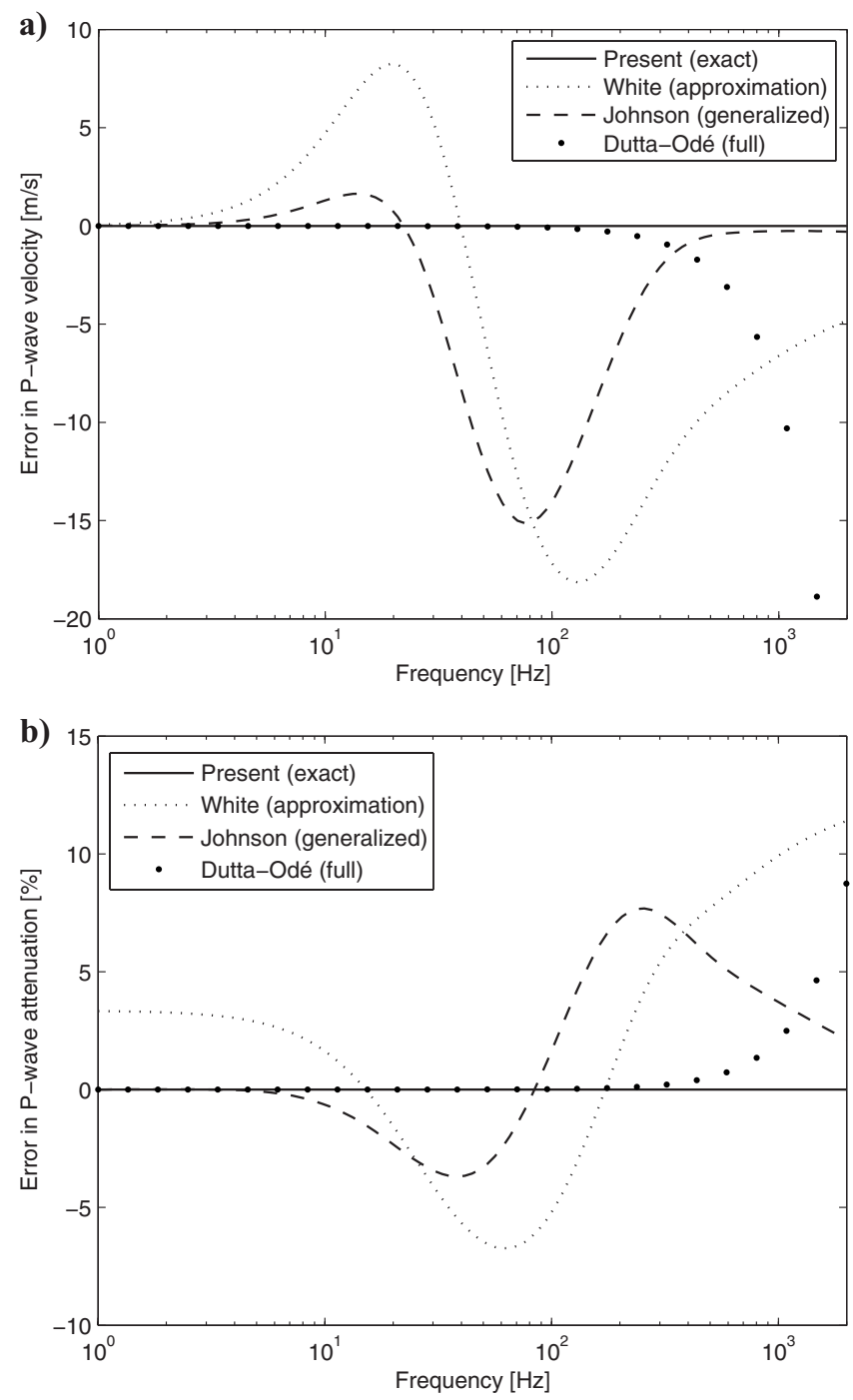

Figure 3. Deviation in the effective $\mathrm{P}$-wave acoustic bulk properties of patchy saturation models for a gas fraction of $10 \%$. (a) Error in phase velocity. (b) Error in attenuation. Legend and input are as in Figure 2. i.e., independent of the physical properties of the gas. Then the static limit for the velocity is again identical to White's static limit, and $K_{\mathrm{BGW}}$ goes to $K_{\mathrm{m}}$. The error in White's low-frequency attenuation, however, remains and is, in fact, exactly the same as before. In the formulation of White (1975), the effective bulk modulus in this specific case still depends on the properties of the gas through his noflow modulus. It is our belief that there is no need to alter the White model because we present here the exact solution to patchy saturation.

Finally, we stress that different patch shapes and distributions in space might show a very different dependency of the bulk modulus, velocity, and attenuation on frequency. The relaxation frequency decreases from regular (periodic) to irregular (e.g., fractal, random) patch distributions (Müller et al., 2008, and references therein), whereas the relaxation frequency increases from simple (e.g., spherical) to complex (e.g., fractal) patch shapes (Picotti et al., 2010, and references therein). In either case, the peak attenuation decreases and the crossover region from the low to the high frequency asymptote becomes broader.
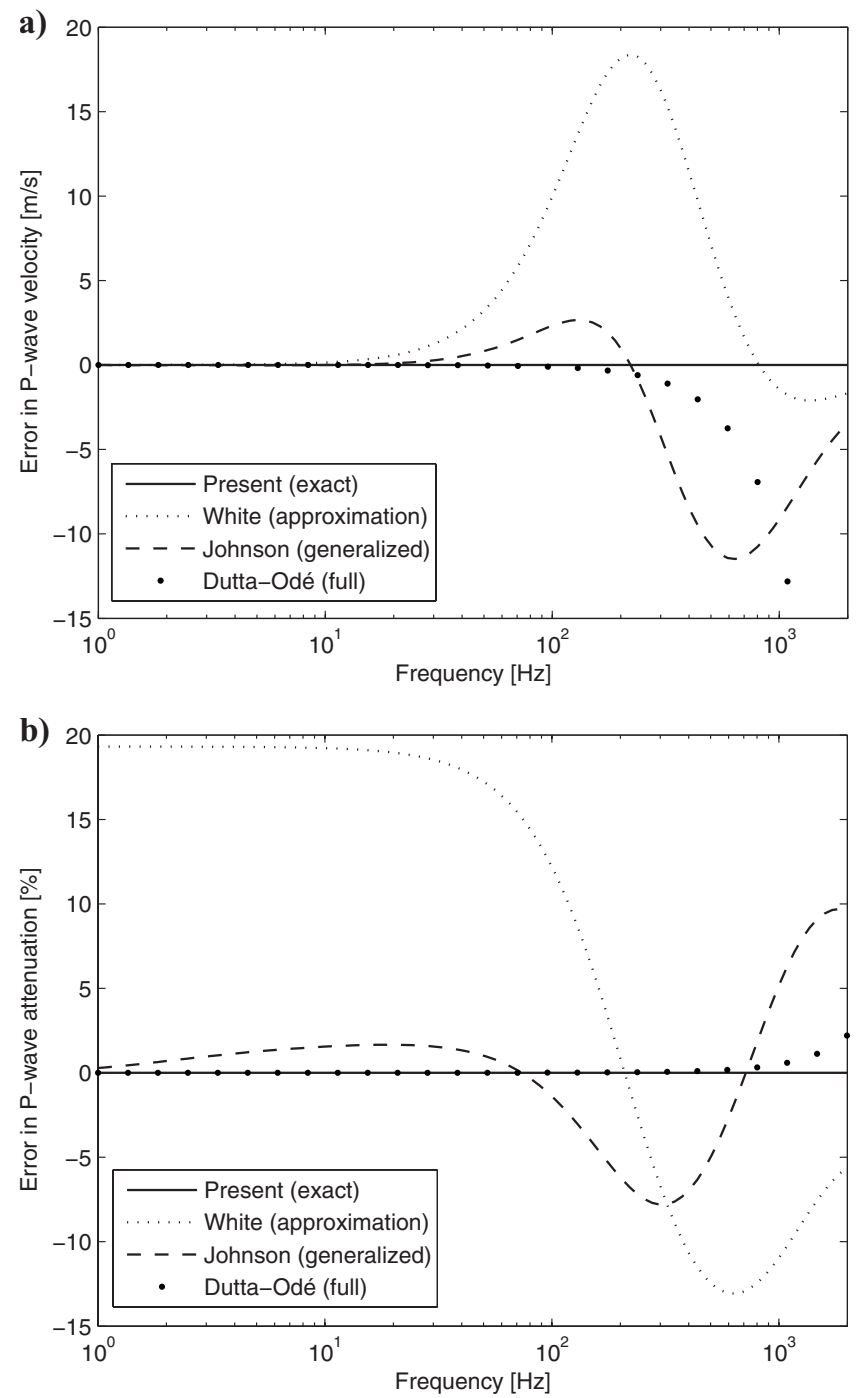

Figure 4. Deviation in the effective P-wave acoustic bulk properties of patchy saturation models for a gas fraction of $50 \%$. (a) Error in phase velocity. (b) Error in attenuation. Legend is as in Figure 2. 
Because we consider only spherical concentric patches in a regular periodic distribution, our simplified model probably is not the best for questions related to the interpretation of patchy-saturated rocks in general. In this respect, the generalized Johnson function might be a better candidate, but for spherical patches our analytic results facilitate rapid comparison with other existing models.

\section{CONCLUSIONS}

We derived an exact analytic expression of the effective bulk modulus for partially saturated media with spherical gas patches. Patchy theories apply to a porous medium fully saturated by a fluid that contains gas pockets larger than the typical pore size. The derivation is based on the quasi-static Biot equations of poroelasticity applied to open-pore boundary conditions.

Our exact analytic solution is identical to the numerical solution as long as the frequency is low enough so that the wavelength of the fast compressional and shear waves are large compared to the dimensions of the pockets and their mutual distance. Unlike the numerical solution, the quasi-static analytic solution does not result in a loss of accuracy from the inversion of ill-conditioned equations for certain input or from the subtraction of large arguments in the spherical Bessel and Neumann functions. Our expression provides the extreme cases of complete liquid or complete gas saturation, as well as the case in which the compressibility of the gas can be neglected. In addition, the expressions for the static and no-flow frequency limit are deduced directly from the quasi-static expression of the effective bulk modulus.

The analytic expression is particularly convenient for computations because it is concise and exact. Such computations allow calculations of the dispersion of the P-wave phase velocity and the level of intrinsic attenuation, and wave-induced fluid flow at the gas-water contact area (relative fluid to solid displacement). We found that the phase velocity and attenuation calculated from the generalized analytic expression of the Johnson model differ as much as $15 \%$ from the exact Johnson numerical solution at seismic frequencies. The errors in the White model are as high as $20 \%$ in the seismic range, and despite the static-velocity corrections, the model does not yield the correct low-frequency level for the P-wave attenuation.

Finally, our analytic expression allows one to find the exact value of the transition frequency at which mesoscopic loss (pressure equilibration of the pore fluids through diffusion) is maximum. Because the production and injection phases of a hydrocarbon reservoir perturb the fluid fractions, knowledge of the exact transition frequency and level of attenuation is of particular interest for such seismic applications as reservoir characterization and time-lapse modeling in tracking fluid movements.

\section{ACKNOWLEDGMENTS}

The work of B.V. was performed with the financial support of Shell and Fundamenteel Onderzoek der Materie (FOM) when at Delft University of Technology and with support of ExxonMobil, Schlumberger, General Electric, and Toyota in the Global Climate and Energy Project (GCEP) when at Stanford University. We thank Jack Dvorkin for reviewing the original draft of the manuscript. We thank three reviewers and the associate editor Tobias Müller for having made useful comments that improved the paper.

\section{APPENDIX A}

\section{STATIC LIMIT}

The static limit of the bulk modulus can be deduced directly from equation 32. For $\omega \rightarrow 0$, we have to use the asymptotic forms of the spherical Bessel functions with small arguments: $\lim _{z \rightarrow 0} j_{0}(z)=1$, $\lim _{z \rightarrow 0} j_{1}(z)=z / 3, \quad \lim _{z \rightarrow 0} n_{0}(z)=-1 / z, \quad$ and $\quad \lim _{z \rightarrow 0} n_{1}(z)=$ $-1 / z^{2}$ (Abramowitz and Stegun, 1965). The static limit of the auxiliary parameter in equation 26 is

$$
\lim _{\omega \rightarrow 0} h=-\frac{\eta_{a}}{\eta_{b}} \frac{D_{a}}{D_{b}} \frac{s_{b}}{s_{a}}
$$

where $s_{b}=1-s_{a}$. We find for equation 29 that

$$
\begin{aligned}
\lim _{\omega \rightarrow 0} g & =s_{a}\left(1-\frac{H_{b}}{H_{a}}-\frac{k_{0}}{\phi^{2}} \frac{s_{b}}{H_{b}} \frac{N^{2}}{s_{a} D_{b} \eta_{b}+s_{b} D_{a} \eta_{a}}\right) \\
& =\frac{s_{a}\left(Q_{b}+R_{b}\right)\left(H_{a}-H_{b}\right)}{s_{a}\left(Q_{b}+R_{b}\right) H_{a}+s_{b}\left(Q_{a}+R_{a}\right) H_{b}} .
\end{aligned}
$$

The zero values of $g$ and $h$ are real-valued. Substituting this result in equation 1 , we have that

$$
\begin{aligned}
\lim _{\omega \rightarrow 0} K(\omega) & \equiv K_{\mathrm{BGW}} \\
& =\frac{K_{\mathrm{BG} b}\left(K_{\mathrm{BG} a}-K_{\mathrm{m}}\right)+s_{a} K_{\mathrm{m}}\left(K_{\mathrm{BG} b}-K_{\mathrm{BG} a}\right)}{\left(K_{\mathrm{BG} a}-K_{\mathrm{m}}\right)+s_{a}\left(K_{\mathrm{BG} b}-K_{\mathrm{BG} a}\right)} .
\end{aligned}
$$

We used

$$
K_{\mathrm{BG}}-\frac{(Q+R)^{2}}{R}=K_{\mathrm{m}} .
$$

Following Johnson (2001), the zero-frequency modulus is called the Biot-Gassmann-Wood modulus $K_{\mathrm{BGW}}$.

A more accessible form of the modulus $K_{\mathrm{BGW}}$ is found if we use the expression for the Biot-Gassmann modulus:

$$
K_{\mathrm{BGW}} \equiv K_{\mathrm{BG}}\left(K_{\mathrm{W}}\right)=K_{\mathrm{m}}+\alpha^{2}\left[\frac{\alpha-\phi}{K_{\mathrm{s}}}+\frac{\phi}{K_{\mathrm{W}}}\right]^{-1} \text {, }
$$

where the fluid modulus $K_{\mathrm{f}}$ is replaced in this case by the harmonic average $K_{\mathrm{W}}$ of the two fluid moduli in regions $a$ and $b$ (Wood's formula):

$$
\frac{1}{K_{\mathrm{W}}}=\frac{s_{a}}{K_{\mathrm{f} a}}+\frac{s_{b}}{K_{\mathrm{f} b}} .
$$

This was discussed earlier by Dutta and Odé (1979b) and Norris (1993) explicitly for layered and spherical patches. Johnson (2001) states that equation A-6 is an exact result, independent of the spatial distribution of the fluids. Using the input of Table 1 with $s_{a}=0.1$, $K_{\mathrm{BGW}}$ is equal to $2.64 \mathrm{GPa}$. It is the lower bound of the effective bulk modulus in Figure 2 and is well approximated by $K_{\mathrm{m}}$ in this case. 
The static limit of the bulk modulus can also be derived from the static limit of the Biot (1956a, 1956b) theory. The Biot equations of motion then reduce to (Dutta and Odé, 1979b)

$$
\begin{aligned}
& \frac{\partial}{\partial r}(\nabla \cdot \mathbf{u})=0, \\
& \frac{\partial}{\partial r}(\nabla \cdot \mathbf{w})=0 .
\end{aligned}
$$

Note that we work here with relative displacement $\mathbf{w}=\phi(\mathbf{U}-\mathbf{u})$. The solutions for spherically symmetrical displacements are

$$
\begin{gathered}
u(r)=A r+B r^{-2}, \\
w(r)=F r+G r^{-2} .
\end{gathered}
$$

Hence, we have for the pore pressure and total stress from equations 5 and 6 that

$$
\begin{gathered}
p=-\frac{Q+R}{\phi}\left(\frac{\partial u}{\partial r}+\frac{2 u}{r}\right)-\frac{R}{\phi^{2}}\left(\frac{\partial w}{\partial r}+\frac{2 w}{r}\right), \\
\tau=H \frac{\partial u}{\partial r}+(H-2 \mu) \frac{2 u}{r}+\frac{Q+R}{\phi}\left(\frac{\partial w}{\partial r}+\frac{2 w}{r}\right),
\end{gathered}
$$

and therefore,

$$
\begin{gathered}
p=-\frac{3(Q+R)}{\phi} A-\frac{3 R}{\phi^{2}} F, \\
\tau(r)=3 K_{\mathrm{BG}} A+\frac{3(Q+R)}{\phi} F-\frac{4 \mu}{r^{3}} B .
\end{gathered}
$$

We note that the pressure is no longer a function of $r$ as a result of the displacement functions $u$ and $w$ specified in equations A-10 and A-11. Again, $Q, R$, and $K_{\mathrm{BG}}$ are elastic constants, and $A, B, F$, and $G$ are unknown variables in both regions. A finite solution at $r=0 \mathrm{im}-$ plies $B_{a}=0$ and $G_{a}=0$. The remaining six unknowns are determined by using the boundary conditions 13-18.

From the continuity of pressure and total stress at the interface, equations 15 and 16 , we have that

$$
\begin{aligned}
& -\frac{3\left(Q_{a}+R_{a}\right)}{\phi} A_{a}-\frac{3 R_{a}}{\phi^{2}} F_{a}=-\frac{3\left(Q_{b}+R_{b}\right)}{\phi} A_{b}-\frac{3 R_{b}}{\phi^{2}} F_{b}, \\
& 3 K_{\mathrm{BG} a} A_{a}+\frac{3\left(Q_{a}+R_{a}\right)}{\phi} F_{a}=3 K_{\mathrm{BG} b} A_{b}+\frac{3\left(Q_{b}+R_{b}\right)}{\phi} F_{b} \\
& -\frac{4 \mu}{r^{3}} B_{b}
\end{aligned}
$$

We note that

$$
\frac{(Q+R) \phi}{R}=1-\frac{K_{\mathrm{m}}}{K_{\mathrm{s}}}=\alpha,
$$

so that this value is identical in both regions because it does not depend on the fluid properties. Multiplication of the left- and righthand sides of equation A-16 by $(Q+R) \phi / R$ and subsequent addition to equation $\mathrm{A}-17$ yields that

$$
K_{\mathrm{m}} A_{a}=K_{\mathrm{m}} A_{b}-\frac{4}{3} \frac{\mu}{a^{3}} B_{b}
$$

where we have used equation A-5. This expression combined with the continuity of solid displacement, $A_{a} a=A_{b} a+B_{b} / a^{2}$, gives

$$
\begin{gathered}
A_{a}=A_{b}, \\
B_{b}=0 .
\end{gathered}
$$

The no-flow condition at the outer boundary, equation 17 , and the continuity of relative displacement at $r=a$, equation 14 , combine to

$$
\begin{gathered}
G_{b}=-F_{b} b^{3}, \\
s_{a} F_{a}=-s_{b} F_{b} .
\end{gathered}
$$

The condition of the external stress at the outer boundary, equation 18 , now yields

$$
3 K_{\mathrm{BG} b} A_{a}-\frac{3 s_{a}}{s_{b}} \frac{Q_{b}+R_{b}}{\phi} F_{a}=-p_{\mathrm{e}} .
$$

By combining with the rewritten equation A-17,

$$
3\left(K_{\mathrm{BG} a}-K_{\mathrm{BG} b}\right) A_{a}+\frac{3}{\phi}\left[Q_{a}+R_{a}+\left(Q_{b}+R_{b}\right) \frac{s_{a}}{s_{b}}\right] F_{a}=0,
$$

we find that

$$
\begin{gathered}
F_{a}=\frac{1}{3} \frac{s_{b}\left(K_{\mathrm{BG} a}-K_{\mathrm{BG} b}\right) \phi}{s_{a}\left(Q_{b}+R_{b}\right) K_{\mathrm{BG} a}+s_{b}\left(Q_{a}+R_{a}\right) K_{\mathrm{BG} b}} p_{\mathrm{e}}, \\
A_{a}=-\frac{1}{3} \frac{s_{a}\left(Q_{b}+R_{b}\right)+s_{b}\left(Q_{a}+R_{a}\right)}{s_{a}\left(Q_{b}+R_{b}\right) K_{\mathrm{BG} a}+s_{b}\left(Q_{a}+R_{a}\right) K_{\mathrm{BG} b}} p_{\mathrm{e}} .
\end{gathered}
$$

Using $u_{b}(b)=A_{b} b$ in equation 1 , we have that the effective bulk modulus in the static limit is given by

$$
\begin{aligned}
K_{\mathrm{BGW}} & =-\frac{b}{3 u_{b}(b)} p_{\mathrm{e}}=-\frac{1}{3 A_{b}} p_{\mathrm{e}} \\
& =\frac{s_{a}\left(K_{\mathrm{BG} b}-K_{\mathrm{m}}\right) K_{\mathrm{BG} a}+s_{b}\left(K_{\mathrm{BG} a}-K_{\mathrm{m}}\right) K_{\mathrm{BG} b}}{s_{a}\left(K_{\mathrm{BG} b}-K_{\mathrm{m}}\right)+s_{b}\left(K_{\mathrm{BG} a}-K_{\mathrm{m}}\right)} .
\end{aligned}
$$

Using $s_{a}+s_{b}=1$, this can be rewritten easily as equation A-4.

Equation A-21 means that in the zero-frequency limit, the total stress is constant throughout both regions and equal to the applied 
external radial stress. The pore pressure also is constant and a fraction of the external radial stress. From equations A-14, A-5, and A-26, we find that this fraction is

$$
\lim _{\omega \rightarrow 0} \frac{p}{p_{\mathrm{e}}}=\frac{1}{\alpha} \frac{\left(K_{\mathrm{BG} a}-K_{\mathrm{m}}\right)\left(K_{\mathrm{BG} b}-K_{\mathrm{m}}\right)}{\left(K_{\mathrm{BG} a}-K_{\mathrm{m}}\right) K_{\mathrm{BG} b}+K_{\mathrm{m}} s_{a}\left(K_{\mathrm{BG} b}-K_{\mathrm{BG} a}\right)},
$$

as also found by Dutta and Odé (1979b).

\section{APPENDIX B}

\section{NO-FLOW LIMIT}

The high-frequency limit can also be deduced directly from equation 32. For $\omega \rightarrow \infty$, the frequency-dependent term in $g$ vanishes, so that

$$
\lim _{\omega \rightarrow \infty} g=s_{a}\left(1-\frac{H_{b}}{H_{a}}\right) .
$$

The no-flow limit of $g$ is real-valued. With equation 1 , it is easy to show that

$$
\lim _{\omega \rightarrow \infty} K(\omega) \equiv K_{\mathrm{BGH}}=\frac{K_{\mathrm{BG} b} H_{a}+\frac{4}{3} \mu s_{a}\left(K_{\mathrm{BG} a}-K_{\mathrm{BG} b}\right)}{H_{a}-s_{a}\left(K_{\mathrm{BG} a}-K_{\mathrm{BG} b}\right)},
$$

where $K_{\mathrm{BGH}}$ is the Biot-Gassmann-Hill modulus. With a uniform frame (i.e., constant $\mu, K_{\mathrm{m}}$, and $K_{\mathrm{s}}$ ), the effective bulk modulus of the medium is in exact agreement with Hill's (1963) theorem:

$$
\frac{1}{K_{\mathrm{BGH}}+\frac{4}{3} \mu}=\frac{s_{a}}{K_{\mathrm{BG} a}+\frac{4}{3} \mu}+\frac{1-s_{a}}{K_{\mathrm{BG} b}+\frac{4}{3} \mu}=\frac{s_{a}}{H_{a}}+\frac{s_{b}}{H_{b}},
$$

as Norris (1993) argued. In our example, $K_{\mathrm{BGH}}=7.39 \mathrm{GPa}$ is the upper bound of the effective bulk modulus in Figure 2.

We also consider the high-frequency limit of $K(\omega)$ under the assumption that the frequency is never so high as to violate $\omega \ll\left(\omega_{\mathrm{B}}, \omega_{r}\right)$. This case is discussed by White (1975) and Dutta and Odé (1979b). As the frequency of the external stress becomes higher, the fluid has little time to flow. In case of the no-flow limit, the equations of motion become

$$
\begin{gathered}
\frac{\partial}{\partial r}(\nabla \cdot \mathbf{u})=0, \\
\mathbf{w}=0,
\end{gathered}
$$

with the solution

$$
u(r)=A r+B r^{-2} .
$$

Therefore (cf. equations A-12 and A-13),

$$
p=-\frac{3(Q+R)}{\phi} A
$$

$$
\tau(r)=3 K_{\mathrm{G}} A-\frac{4 \mu}{r^{3}} B .
$$

Again, $B_{a}=0$, and the pore pressure is no longer a function of $r$. Three boundary conditions 13,16 , and 18 provide expressions for $A_{a}, A_{b}$, and $B_{b}$.

The continuity of total stress and solid displacement at the inner boundary yields that

$$
A_{a}=A_{b}+\frac{B_{b}}{a^{3}}
$$

$$
B_{b}=-A_{b} a^{3} \frac{K_{\mathrm{BG} a}-K_{\mathrm{BG} b}}{H_{a}} .
$$

Substitution of the above in the total stress condition at the outer boundary yields that

$$
A_{b}=-\frac{p_{\mathrm{e}}}{3 K_{\mathrm{BG} b}+4 \mu s_{a}\left(K_{\mathrm{BG} a}-K_{\mathrm{BG} b}\right) / H_{a}},
$$

so that the effective bulk modulus in the high-frequency limit is given by equation B- 2 .

We realize that although the pore pressure is constant within each phase, it is discontinuous at the inner boundary. Equations A-5, B-7, $\mathrm{B}-9$, and $\mathrm{B}-11$ then give

$$
\lim _{\omega \rightarrow \infty} \frac{p_{a}}{p_{\mathrm{e}}}=\frac{\left(K_{\mathrm{BG} a}-K_{\mathrm{m}}\right) H_{b}}{\alpha\left(K_{\mathrm{BG} b} H_{a}+\frac{4}{3} \mu s_{a}\left(K_{\mathrm{BG} a}-K_{\mathrm{BG} b}\right)\right)},
$$

$$
\lim _{\omega \rightarrow \infty} \frac{p_{b}}{p_{\mathrm{e}}}=\frac{\left(K_{\mathrm{BG} b}-K_{\mathrm{m}}\right) H_{a}}{\alpha\left(K_{\mathrm{BG} b} H_{a}+\frac{4}{3} \mu s_{a}\left(K_{\mathrm{BG} a}-K_{\mathrm{BG} b}\right)\right)} .
$$

This discontinuity at the inner boundary is due to the different physical properties of the fluids in each region. However, the total radial bulk stress is continuous, and because there is no relative fluid-flow, the inner boundary acts as if it were sealed.

\section{REFERENCES}

Abramowitz, M., and I. A. Stegun, 1965, Handbook of mathematical functions with formulas, graphs, and mathematical tables: Dover Publications. Berryman, J. G., and L. Thigpen, 1985, Effective constants for wave propagation through partially saturated porous media: Applied Physics Letters, 46, 722-724.

Biot, M. A., 1956a, Theory of propagation of elastic waves in a fluid-saturated porous solid: Part 1 - Low frequency range: Journal of the Acoustical Society of America, 28, 168-178.

$-1956 \mathrm{~b}$, Theory of propagation of elastic waves in a fluid-saturated porous solid: Part 2 - High frequency range: Journal of the Acoustical Society of America, 28, 179-191.

Biot, M. A., and D. G. Willis, 1957, The elastic coefficients of the theory of consolidation: Journal of Applied Mechanics, 24, 594-601.

Cadoret, T., D. Marion, and B. Zinszner, 1995, Influence of frequency and fluid distribution on elastic-wave velocities in partially saturated limestones: Journal of Geophysical Research — Solid Earth, 100,9789-9803.

Carcione, J. M., H. B. Helle, and N. H. Pham, 2003, White's model for wave propagation in partially saturated rocks: Comparison with poroelastic numerical experiments: Geophysics, 68, 1389-1398. 
Castagna, J. P., S. Sun, and R. W. Seigfried, 2003, Instantaneous spectral analysis: Detection of low-frequency shadows associated with hydrocarbons: The Leading Edge, 22, 120-127.

Chapman, M., E. R. Liu, and X. Y. Li, 2006, The influence of fluid-sensitive dispersion and attenuation on AVO analysis: Geophysical Journal International, 167, 89-105.

Ciz, R., and B. Gurevich, 2005, Amplitude of Biot's slow wave scattered by a spherical inclusion in a fluid-saturated poroelastic medium: Geophysical Journal International, 160, 991-1005.

Deresiewicz, H., and R. Skalak, 1963, On uniqueness in dynamic poroelasticity: Bulletin of the Seismological Society of America, 53, 783-788.

Dutta, N. C., and H. Odé, 1979a, Attenuation and dispersion of compressional waves in fluid-filled porous rocks with partial gas saturation (White model): Part 1 - Biot theory: Geophysics, 44, 1777-1788.

-, 1979b, Attenuation and dispersion of compressional waves in fluidfilled porous rocks with partial gas saturation (White model): Part $2-\mathrm{Re}-$ sults: Geophysics, 44, 1789-1805.

Dutta, N. C., and A. J. Seriff, 1979, White model of attenuation in rocks with partial gas saturation: Geophysics, 44, 1806-1812.

Gelinsky, S., S. A. Shapiro, T. M. Müller, and B. Gurevich, 1998, Dynamic poroelasticity of thinly layered structures: International Journal of Solids and Structures, 35, 4739-4751.

Goloshubin, G. M., C. van Schuyver, V. A. Korneev, D. B. Silin, and V. Vingalov, 2006, Reservoir imaging using low frequencies of seismic reflections: The Leading Edge, 25, 527-531.

Gurevich, B., M. Brajanovski, R. Galvin, T. M. Müller, and J. Toms-Stewart, 2009, P-wave dispersion and attenuation in fractured and porous reservoirs - Poroelasticity approach: Geophysical Prospecting, 57, 225-237.

Gurevich, B., and S. L. Lopatnikov, 1995, Velocity and attenuation of elasticwaves in finely layered porous rocks: Geophysical Journal International, 121, 933-947.

Helle, H. B., N. H. Pham, and J. M. Carcione, 2003, Velocity and attenuation in partially saturated rocks: Poroelastic numerical experiments: Geophysical Prospecting, 51, 551-566.

Hill, R., 1963, Elastic properties of reinforced solids - Some theoretical principles: Journal of the Mechanics and Physics of Solids, 11, 357-372.

Jocker, J., D. M. J. Smeulders, G. G. Drijkoningen, C. van der Lee, and A. Kalfsbeek, 2004, Matrix propagator method for layered porous media: Analytical expressions and stability criteria: Geophysics, 69, 1071-1081.

Johnson, D. L., 2001, Theory of frequency dependent acoustics in patchysaturated porous media: Journal of the Acoustical Society of America, 110, 682-694.

Knight, R. J., J. Dvorkin, and A. Nur, 1998, Acoustic signatures of partial saturation: Geophysics, 63, 132-138.

Lebedev, M., J. Toms, B. Clennell, M. Pervukhina, V. Shulakova, L. Paterson, T. M. Müller, B. Gurevich, and F. Wenzlau, 2009, Direct laboratory observation of patchy saturation and its effects on ultrasonic velocities: The Leading Edge, 28, 24-27.

Lee, M. W., and T. S. Collett, 2009, Unique problems associated with seismic analysis of partially gas-saturated unconsolidated sediments: Marine and Petroleum Geology, 26, 775-781.

Masson, Y. J., and S. R. Pride, 2007, Poroelastic finite difference modeling of seismic attenuation and dispersion due to mesoscopic-scale heterogeneity: Journal of Geophysical Research, 112, B03204.

Mavko, G. M., and T. Mukerji, 1998, Bounds on low-frequency seismic velocities in partially saturated rocks: Geophysics, 63, 918-924.

Monsen, K., and S. Johnstad, 2005, Improved understanding of velocity-saturation relationships using 4D computer-tomography acoustic measurements: Geophysical Prospecting, 53, 173-181.

Morse, P. M., and K. U. Ingard, 1968, Theoretical acoustics: Princeton University Press.
Müller, T. M., and B. Gurevich, 2004, One-dimensional random patchy saturation model for velocity and attenuation in porous rocks: Geophysics, 69 , $1166-1172$

, 2005, Wave-induced fluid flow in random porous media: Attenuation and dispersion of elastic waves: Journal of the Acoustical Society of America, 117, 2732-2741.

Müller, T. M., J. Toms-Stewart, and F. Wenzlau, 2008, Velocity-saturation relation for partially saturated rocks with fractal pore fluid distribution: Geophysical Research Letters, 35, L09306.

Murphy, W. F., 1982, Effects of partial water saturation on attenuation in Massilon sandstone and Vycor porous glass: Journal of the Acoustical Society of America, 71, 1458-1468.

Norris, A. N., 1993, Low-frequency dispersion and attenuation in partially saturated rocks: Journal of the Acoustical Society of America, 94, 359-370.

Paffenholz, J., and H. Burkhardt, 1989, Absorption and modulus measurements in the seismic frequency and strain range on partially saturated sedimentary rocks: Journal of Geophysical Research, 94, 9493-9507.

Picotti, S., J. M. Carcione, J. G. Rubino, and J. E. Santos, 2007, P-wave seismic attenuation by slow-wave diffusion: Numerical experiments in partially saturated rocks: Geophysics, 72, no. 4, N11-N21.

Picotti, S., J. M. Carcione, J. G. Rubino, J. E. Santos, and F. Cavallini, 2010 A viscoelastic representation of wave attenuation in porous media: Computers \& Geosciences, 36, 44-53.

Pride, S. R., J. G. Berryman, and J. M. Harris, 2004, Seismic attenuation due to wave-induced flow: Journal of Geophysical Research, 109, B01201.01B01201.19.

Pride, S. R., E. Tromeur, and J. G. Berryman, 2002, Biot slow-wave effects in stratified rock: Geophysics, 67, 271-281.

Quintal, B., S. M. Schmalholz, and Y. Y. Podladchikov, 2009, Low-frequency reflections from a thin layer with high attenuation caused by interlaye flow: Geophysics, 74, no. 1, N15-N23.

Saenger, E. H., S. Schmalholz, M. Lambert, T. Nguyen, A. Torres, S. Metzger, R. Habiger, T. Muller, S. Rentsch, and E. Mendez-Hernandez, 2009, A passive seismic survey over a gas field: Analysis of low-frequency anomalies: Geophysics, 74, no. 2, O29-O40.

Shapiro, S. A., and T. M. Müller, 1999, Seismic signatures of permeability in heterogeneous porous media: Geophysics, 64, 99-103.

Taylor, S. R., and R. J. Knight, 2003, An inclusion-based model of elastic wave velocities incorporating patch-scale fluid pressure relaxation: Geophysics, 68, 1503-1509.

Toms, J., T. M. Müller, R. Ciz, and B. Gurevich, 2006, Comparative review of theoretical models for elastic wave attenuation and dispersion in partially saturated rocks: Soil Dynamics and Earthquake Engineering, 26, $548-565$.

Toms-Stewart, J., T. M. Müller, B. Gurevich, and L. Paterson, 2009, Statistical characterization of gas-patch distributions in partially saturated rocks Geophysics, 74, no. 2, WA51-WA64.

Vogelaar, B. B. S. A., and D. M. J. Smeulders, 2007, Extension of White's layered model to the full frequency range: Geophysical Prospecting, $\mathbf{5 5}$ 685-695.

Wenzlau, F., and T. M. Müller, 2009, Finite-difference modeling of wave propagation and diffusion in poroelastic media: Geophysics, 74, no. 4, T55-T66.

White, J. E., 1975, Computed seismic speeds and attenuation in rocks with partial gas saturation: Geophysics, 40, 224-232.

White, J. E., N. Mikhaylova, and F. Lyakhovitskiy, 1975, Low-frequency seismic waves in fluid-saturated layered rocks: Izvestija Academy of Sciences USSR, Physics of Solid Earth, 11, 654-659.

Winkler, K. W., and A. Nur, 1979, Pore fluids and seismic attenuation in rocks: Geophysical Research Letters, 6, 1-4. 